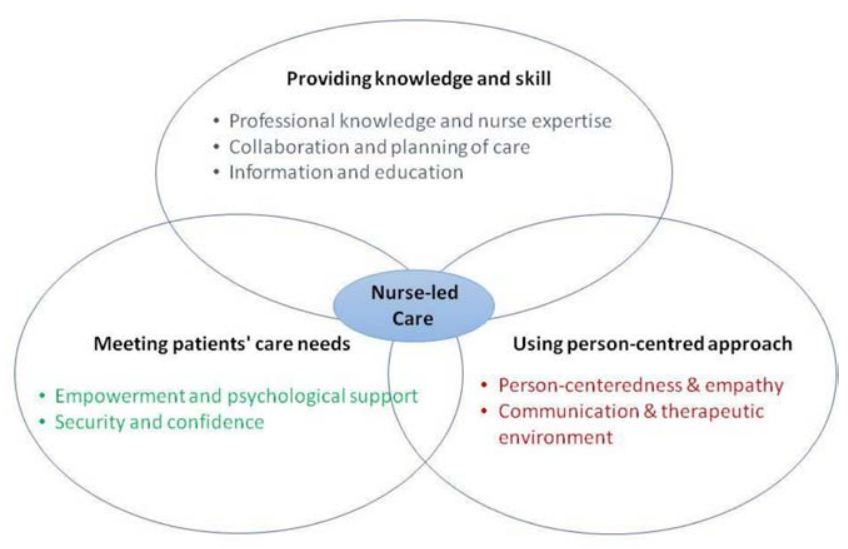

Figure 1. Themes of nurse-led care from the perspective of people with RA

Providing knowledge and skill. This theme delineated rheumatology nursing as providing professional expertise in the planning and delivery of care. The rheumatology nurse-led service included easy access via telephone helpline, consultations with the clinical nurse specialist for assessment of disease activity and care needs, planning of care, disease information and education, supporting self-management, and referral to rheumatologist and the multi-disciplinary team. People with RA highly valued the nurse expertise and specialist knowledge provided at nurse-led clinics. 'She was very good at informing me, so I have only praise for this ... because I have never had it like this before'. (Person with early $R A)$.

Using a person-centred approach. This theme showed nurse-led care using a person-centred approach combined with empathy and good communication skills, which created a good therapeutic environment. People with RA appreciated the person-centeredness, empathy and involvement of the nurse. 'She is very sensitive. She can see if I am feeling bad and comes straight to me and asks: "How are you today?"...You are treated and taken seriously. (Person with early RA).

Meeting patients' care needs. This theme presented nurse-led care as creating a sense of being empowered and psychologically supported in the management of RA and its impact. Nurse-led care made people with RA feel cared for, secure and confident. It added value to rheumatology care and made care complete. 'The thought of sticking a needle into my own stomach... it felt a bit like I would never manage to do that. However, they have been absolutely wonderful here ... and now I can do it myself'. (Person with early RA).

Conclusion: Nurse-led care for people with RA is characterised by provision of rheumatology expertise using a person-centred approach, and patients' holistic care needs are being met. This study found a dearth of literature on perceptions of nurse-led care in people with early RA, which highlights the need for further research in this population.

References:

[1] Thomas J, Harden A. Methods for the thematic synthesis of qualitative research in systematic reviews. BMC Med Res Methodol 2008; 8: 45 .

Disclosure of Interests: Anne-Marie Tetsche Sweeney: None declared, Candy McCabe: None declared, Caroline Flurey: None declared, Joanna Robson: None declared, Alice Berry: None declared, Pamela Richards: None declared, Mwidimi Ndosi Grant/research support from: Bristol Myers Squibb, Consultant of: Janssen, Pfizer

DOI: 10.1136/annrheumdis-2020-eular.502

\section{SAT0644-HPR COMPLIANCE OF BIOLOGIC DISEASES MODIFYING ANTI-RHEUMATIC DRUGS (BDMARDS) WITH SYSTEMIC IMMUNO-INFLAMMATORY RHEUMATIC DISEASES (SIRDS). AN ASSESSMENT OF PATIENTS ADHERENCE AND NON- ADHERENCE CONCERNS.}

R. Thakran ${ }^{1}$, S. Baghel' ${ }^{1}$, C. Messi ${ }^{1}$, V. Kumar' ${ }^{1}$, S. Kapoor ${ }^{1}$, S. Garg ${ }^{1}$, A. Malaviya ${ }^{1}$ on behalf of no. ${ }^{1}$ Indian Spinal Injuries Centre, rheumatology, New Delhi, India

Background: Patients with systemic immunoinflammatory rheumatic diseases (SIRDs) are often treated with bDMARDs when the response to conventional disease-modifying antirheumatic drugs (csDMARDs) is inadequate.There are, however, concerns about non-adherence to bDMARDs among patient. The non-adherence to bDMARDs may be caused by the various factors.

Objectives: 1.The main objective of present study was to find out the cause of discontinuation of bDMARDs

2.To find out the adherence and non-adherence rate for bDMARDs.

3.To identify the factors that are modifiable.
Methods: $\mathbf{8 0 0}$ patients with SIRDs prescribed bDMARDs were interviewed to find out the demographic information, their socioeconomic status, and the disease duration.Additional information gathered included the comorbidities, the time for starting bDMARDs, the route of administration of bDMARDs, beliefs and perceptions about treatment efficacy and side effects if any.This was followed by looking at the adherence of bDMARDs; if they had discontinued then efforts was made to find out the reasons for the same.

Based on these findings the patients were classified into adherent and non-adherent categories. The data were analyzed further for

1.Factors that associated with persistence of bDMARDs.

2.Factors that were associated with discontinuation of bDMARDs.

Results: A total of 800 patients were interviewed that included patients with ankylosing spondylitis $430(52.4 \%)$, rheumatoid arthritis $300(37.7 \%)$, psoriatic arthritis $45(5.2 \%)$, and others $25(0.7 \%)$.On analysis $610(76 \%)$ patient were compliant but $190(24 \%)$ patient had discontinued the bDMARDs on their own On comparison of both groups

Factors that were significantly related to self-discontinuation were:

- Negative beliefs about biologics (37\%)

- Cost (33\%)

- Reading side-effect profile on Google search (25\%)

- Other co-morbidities (6\%)

Factors that were significantly related to persistence of biologic treatment were:

- Good counseling by rheumatologist and rheumatology nurse (60\%)

- Faith in the treating rheumatologist $(25 \%)$

- Fear of deformities and pain(15\%)

On analysis it was found that a good counseling and clarifying the doubts of the patients regarding bDMARDs before starting the treatment encourages the patient to continue the biologic treatment, especially it allays their doubts about the drug adverse effects.

Conclusion: Despite negative beliefs and misconceptions about bDMARDs, patient non-adherence at our center is not alarming.A positive reinforcement counseling appears to be the most significant factor to overcome the negative belief of patients. The affordability of the biologic treatment however remains a limiting factor in our centre as in other parts of India.

References:

[1] Tamas Koncz,MD,Marta,Pentek,Valentin,Brodszky,Katalin Ersek,MSc Ewaorlewska\&Laszlo Gulasi Volume10,2010 -Issue9 Adherence to biologic DMARD therapies in rheumatoid arthritis

Acknowledgments: no

Disclosure of Interests: None declared

DOI: 10.1136/annrheumdis-2020-eular.3026

\section{SAT0645-HPR MYTHS AND MISCONCEPTION ABOUT THE ILLNESS AND CONVENTIONAL SYNTHETIC DMARDS (CSDMARDS) IN PATIENTS WITH SYSTEMIC IMMUNO- INFLAMMATORY RHEUMATIC DISEASES (SIRDS): A STUDY BY RHEUMATOLOGY NURSE COUNSELOR}

R. Thakran ${ }^{1,2}$, S. Baghel ${ }^{1,3}$, L. Khurshid ${ }^{2}$, S. Kapoor4, S. Garg4, A. Malaviya4on behalf of no. ${ }^{1}$ Indian Spinal Injuries Centre, New Delhi, India; ${ }^{2}$ Indian Spinal Injuries Centre, rheumatology, New Delhi, India; ${ }^{3}$ indian spinal injuries centre, rheumatology, New Delhi, India; ${ }^{1}$ Indian Spinal Injuries Centre, New Delhi, India

Background: Myths and misconceptions about illness and conventional disease modifying anti-rheumatic drugs directly influence adherence to the prescribed treatment. It is estimated that $30-50 \%$ of patients do not adhere to their prescribed treatment due to various reasons where the beliefs of the patients play a crucial role. At our centre we the specialist rheumatology nurse counsel the patients at every visit and try to remove their myths and negative beliefs about the disease as well as the medications.

Objectives: - To explore the common myths and misconceptions of regarding their disease and regarding the csDMARDs.

- To assess the efficacy of counseling in allaying their unfounded fear.

Methods: A total of 450 patients with SIRDs at least 3 times attended the rheumatology out-patient clinic on csDMARDs were enrolled to complete a questionnaire that, besides demographic information, socio-economic status, and co- morbidities, had the following questions:

1. Self reported adherence to medication

2. Misbelieves regarding food items

3. What kind of health-provider was consulted at the onset of the symptoms

4. Their belief/knowledge regarding:

A. The need for physiotherapy.

B. Life style modification requirement

C. About osteoarthritis

D. Medication requirement during remission

E. Pregnancy and DMARDs

$F$. The need of vaccination 
G. Health hazards of smoking and alcohol use

$\mathrm{H}$. Harms of discontinuing treatment when they felt well

Results: A total of 450 patients included spondyloarthropathy $150(34 \%)$, rheumatoid arthritis $200(45.7 \%)$, psoriatic arthritis $45(10 \%)$, and others $25(5.5 \%)$. The following observations was made:

1.Self-reported adherence to medication was in 250 (55\%) patients; 200(45\%) patient were non-adherent to treatment

$2.382 / 450(85 \%)$ patients had misbelieves regarding different food items.

$3.225 / 450(50) \%$ of the patients were not doing regular physiotherapy they were totally dependent on medications for symptoms relief.

$4.387 / 450(86 \%)$ patients confused the symptoms of osteoarthritis with that of RA.

$5.315 / 450(70 \%)$ patients did not feel the requirement of continuing drugs during remission.

$6.135 / 450(30 \%)$ patients believed that while on DMARDs they cannot contemplate pregnancy.

$7.351 / 450(78 \%)$ patients accept the need for vaccination when staring DMARDs $8.360 / 450$ (80\%) patients aware about side effect of smoking in disease but only $40 \%$ were able to quit.

$9.273 / 450(60 \%)$ patients felt that more expensive medicines e.g.bDMARDs have more effects.

$10.360 / 450(80 \%)$ patients believed that DMARDs were 'steroids' and they increased weight. On analysis one patient have more than two myths simultaneously.

Conclusion: Increased awareness of the patient's beliefs about medicines is needed among health care providers. We should encourage patients to express their views about medicines as well as disease in order to optimize and personalize the information process. This can stimulate concordance and adherence to medication and follow up.These myths are deeply rooted in our society, single sitting counseling is not enough, and reinforcement is needed.

References:

[1] Tom Greenhalgh. Facts about rheumatoid arthritis: 7 myths you may encounter. Rheumatology Advisor. March 28, 2019.

Acknowledgments: no

Disclosure of Interests: None declared

DOI: 10.1136/annrheumdis-2020-eular.3099

\section{SAT0646-HPR PATTERN AND INFLUENTIAL FACTORS IN PROMOTING TREAT-TO-TARGET (T2T) FOR FOLLOW-UP OF ANKYLOSING SPONDYLITIS (AS) PATIENTS WITH A RHEUMATOLOGIST-PATIENT INTERACTIVE SMART SYSTEM OF DISEASE MANAGEMENT (SSDM): A COHORT STUDY FROM CHINA}

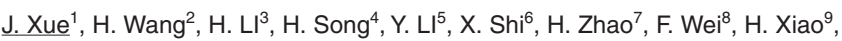
B. Wu ${ }^{9}$, Y. Jia ${ }^{9}, \mathrm{~F} \mathrm{Xiao}^{9}, \mathrm{H}$. Wu $\mathrm{Wu}^{1}$ on behalf of SSDM Collaboration Group, China. ${ }^{1}$ The Second Affiliated Hospital of Zhejiang University school of Medicine, Hangzhou, China; ${ }^{2}$ The First Hospital of Jiaxing, Jiaxing, China; ${ }^{3}$ The Affiliated Hospital of Inner Mongolia Medical University, Neimenggu, China; ${ }^{4}$ Beijing Jishuitan Hospital, Beijing, China; ${ }^{5}$ Zhejiang Provincial People's Hospital, Hangzhou, China; ${ }^{6}$ The First Affiliated Hospital of Henan University of science and technology, Luoyang, China; ${ }^{7}$ Xiangya Hospital of Central South University, Changsha, China; ${ }^{8}$ Jiangmen Central Hospital Affiliated Jiangmen Hospital of Sun Yat-Sen University, Jiangmen, China; ${ }^{9}$ Shanghai Gothic Internet Technology Co., Ltd., Shanghai, China

Background: Ankylosing Spondylitis Disease Activity Score (ASDAS) is adopted to evaluate the degree of disease activity and the inflammatory response in AS patients. ASDAS score <= 1.3 represents inactive disease status and achievement of T2T. SSDM is a mobile application for disease management.

Objectives: To evaluate the patterns of T2T and related influential factors among AS patients after applying SSDM in the real world.

Methods: AS Patients were trained to master SSDM by healthcare professionals (HCPs) and to conduct ASDAS self-assessments. Patients were also required for repeating self-assessments after leaving the hospital. After entry by patients, data can be synchronized to the SSDM terminal of authorized rheumatologists. Based on these data, the patients can apply for consultation to their physicians and rheumatologists can provide medical advices to their patients.

Results: From Jan 2015 to Jan 2020, 17,870 AS patients enrolled in SSDM with the mean age of $34.62 \pm 10.98$ years old and the median disease duration of 3.58 years. Among them, 1,127 AS patients from 150 hospitals were followed up for more than 6 months through SSDM. The results at baseline and in final follow up were summarized in Table 1.
Table 1. The T2T results at baseline and in final follow up.

\begin{tabular}{lcccccccccc}
\hline $\begin{array}{l}\text { BaselinelFinal } \\
\text { follow-up }\end{array}$ & $n$ & $\%$ & $\begin{array}{c}\mathrm{x}<= \\
1.3\end{array}$ & $\begin{array}{l}1.3<\mathrm{x} \\
<=2.1\end{array}$ & $\begin{array}{l}2.1<\mathrm{x} \\
<=3.5\end{array}$ & $\begin{array}{l}3.5 \\
<\mathrm{x}\end{array}$ & $\%$ \\
\hline $\mathrm{x}<=1.3$ & 315 & $27.95 \%$ & 206 & $65.40 \%$ & 74 & $23.49 \%$ & 26 & $8.25 \%$ & 9 & $2.86 \%$ \\
$1.3<\mathrm{x}<=2.1$ & 340 & $30.17 \%$ & 138 & $40.59 \%$ & 114 & $33.53 \%$ & 75 & $22.06 \%$ & 13 & $3.82 \%$ \\
$2.1<\mathrm{x}<=3.5$ & 363 & $32.21 \%$ & 95 & $26.17 \%$ & 106 & $29.20 \%$ & 133 & $36.64 \%$ & 29 & $7.99 \%$ \\
$3.5<\mathrm{x}$ & 109 & $9.67 \%$ & 24 & $22.02 \%$ & 25 & $22.94 \%$ & 42 & $38.53 \%$ & 18 & $16.51 \%$ \\
Total & 1,127 & $100 \%$ & 463 & $41.08 \%$ & 319 & $28.31 \%$ & 276 & $24.49 \%$ & 69 & $6.12 \%$ \\
\hline
\end{tabular}

The rate of T2T achievers were $27.95 \%(315 / 1,127)$ at baseline, and improved significantly to $41.08 \%(463 / 1,127)$ after 6 months follow up, $\mathrm{p}<0.01$. Among T2T achievers at baseline, 65.40\% (206/315) maintained T2T, 34.60\% (109/315) relapsed. Of patients who didn't achieve T2T at baseline, only $31.65 \%(257 / 812)$ achieved T2T after 6 months follow up.

The impact of the online interaction between patients and physicians and the frequency of self-assessment for ASDAS on T2T has been analyzed. Compared with 544 patients who didn't interact online with their physicians and self-assessed less than 3times, 104 patients with online interaction and monthly assessments achieved significant higher improvement rate of T2T $(9.19 \%$ vs $23.08 \%$, $\mathrm{p}<0.01$ ). The more frequent of the self-assessments being performed by patients, the higher improvement of T2T rate will be. The improvement of T2T rate(y) was positively correlated with times of self-assessment for ASDAS(x) independently. The regression equation as " $y=0.0304 x+0.0521$ ", $r=0.9107, p<0.01$ (Figure 1).

Figur1. The correlation between the improvement of T2T rate(y) and the times of self-assessment for ASDAS $(x)$

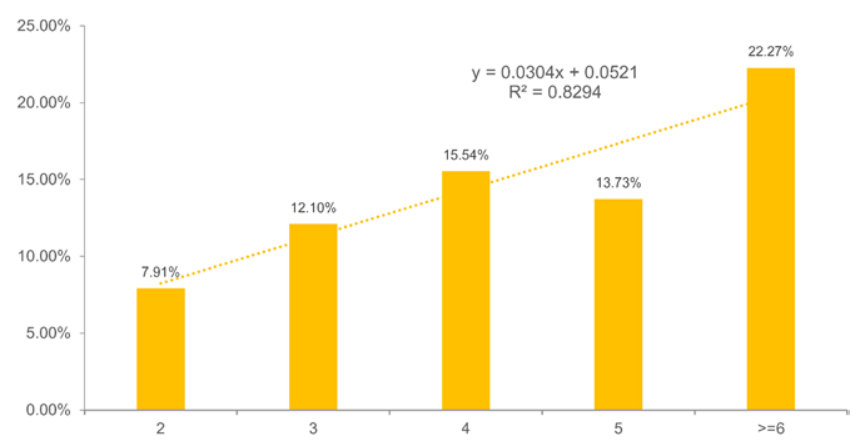

Figure 1.

Conclusion: Significant improvement was observed under applying SSDM through empowering AS patients. After proactive disease management via SSDM for more than 6 months, patients with ASDAS $<=1.3$ score at baseline had a significantly higher retention rate of inactive disease activity. The patients who performed more frequent self-assessments had lower probability of relapse and higher rate of T2T. Online interaction between patients and physicians contributed to promote the improvement rate of T2T. SSDM is a valuable tool for long term follow-up through empowering patients.

Acknowledgments: SSDM was developed by Shanghai Gothic Internet Technology Co., Ltd.

Disclosure of Interests: None declared

DOI: 10.1136/annrheumdis-2020-eular.1798

\section{SAT0647-HPR DEVELOP A MACHINE LEARNING MODEL AND ALGORITHM BASED ON SMART SYSTEM OF DISEASE MANAGEMENT (SSDM) BIG DATA FOR RA FLARE PREDICTION}

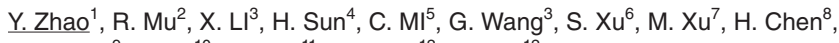
Q. Huang ${ }^{9}$, L. Lei ${ }^{10}$, S. Haili ${ }^{11}$, X. Chen ${ }^{12}$, F. Xiao ${ }^{12}$ on behalf of SSDM Collaboration Group, China. ${ }^{1}$ Peking Union Medical College Hospital, Beijing, China; ${ }^{2}$ People's Hospital, Beijing University Medical School, Beijing, China; ${ }^{3}$ the First Affiliated Hospital of University of Science and Technology of China, Hefei, China; ${ }^{4}$ Shandong Provincial Hospital Affiliated to Shandong University, Jinan, China; ${ }^{5}$ The Second Affiliated Hospital of Guangxi Medical University, Nanning, China; ${ }^{6}$ the First Affiliated Hospital of Anhui Medical University, Hefei, China; ${ }^{7}$ Affiliated Hospital of Hebei University, Baoding, China; ${ }^{8}$ The Third Affiliated Hospital of Hebei Medical University, Shijiazhuang, China; ${ }^{9}$ Guangdong Provincial Hospital of Chinese Medicine, Guangzhou, China;

${ }^{10}$ The First Affiliated Hospital of Guangxi Medical University, Nanning, China;

${ }^{11}$ Second Hospital of Lanzhou University, Lanzhou, China; ${ }^{12}$ Shanghai Gothic Internet Technology Co., Ltd., Shanghai, China 